\title{
QCD factorization and CP asymmetries in hadronic B decays
}

\author{
Matthias Neubert ${ }^{\text {a }}$

 \\ Ithaca, New York 14853, U.S.A.
}

\begin{abstract}
We review recent advances in the theory of strong-interaction effects and final-state interactions in hadronic weak decays of heavy mesons. In the heavy-quark limit, the amplitudes for most nonleptonic, two-body $B$ decays can be calculated from first principles and expressed in terms of semileptonic form factors and lightcone distribution amplitudes. We summarize the main features of this novel QCD factorization and discuss its phenomenological applications to $B \rightarrow D^{(*)} L$ decays (with $L$ a light meson), and to the rare charmless decays $B \rightarrow \pi K$ and $B \rightarrow \pi \pi$.
\end{abstract}

\section{INTRODUCTION}

The theoretical description of hadronic weak decays is difficult due to nonperturbative stronginteraction dynamics. This affects the interpretation of data collected at the $B$ factories, and in many cases limits our ability to uncover the origin of $\mathrm{CP}$ violation and search for New Physics. The complexity of the problem is illustrated in Fig. 1.

It is well known how to control the effects of hard gluons with virtuality between the electroweak scale $M_{W}$ and the scale $m_{B}$ characteristic to the decays of interest. They can be dealt with by constructing a low-energy effective weak Hamiltonian

$\mathcal{H}_{\mathrm{eff}}=\frac{G_{F}}{\sqrt{2}} \sum_{i} \lambda_{i}^{\mathrm{CKM}} C_{i}\left(M_{W} / \mu\right) O_{i}(\mu)+$ h.c.,

where $\lambda_{i}^{\mathrm{CKM}}$ are products of CKM matrix elements, $C_{i}\left(M_{W} / \mu\right)$ are calculable short-distance coefficients, and $O_{i}(\mu)$ are local operators renormalized at a scale $\mu=\mathcal{O}\left(m_{B}\right)$. The challenge is to calculate the hadronic matrix elements of these operators with controlled theoretical uncertainties, using a systematic approximation scheme.

Previous field-theoretic attempts to evaluate these matrix elements have employed dynamical schemes such as lattice field theory, QCD sum rules, or the hard-scattering approach. The first two have difficulties in accounting for finalstate rescattering, which however is important for predicting direct $\mathrm{CP}$ asymmetries. The hard-

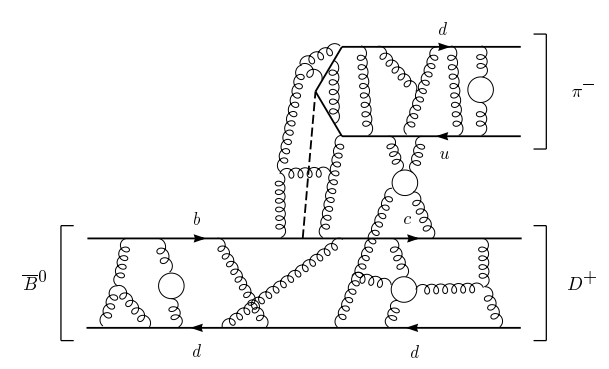

Figure 1. QCD effects in a hadronic decay.

scattering approach misses the leading soft contribution to the $B \rightarrow$ meson transition form factors and thus falls short of reproducing the correct magnitude of the decay amplitudes. In view of these difficulties, most previous analyses of hadronic decays have employed phenomenological models such as "naive" or "generalized factorization", in which the complicated matrix elements of four-quark operators in the effective weak Hamiltonian are replaced, in an ad hoc way, by products of current matrix elements. Corrections to this approximation are accounted for by introducing a set of phenomenological parameters $a_{i}$. A different strategy is to classify nonleptonic decay amplitudes according to flavor topologies ("trees" and "penguins"), which can be decomposed into $\mathrm{SU}(3)$ or isospin amplitudes. This leads to relations between decay amplitudes in the flavor-symmetry limit. No attempt is made to compute these amplitudes from first principles. 


\section{QCD FACTORIZATION FORMULA}

Here we summarize recent progress in the theoretical understanding of nonleptonic decay amplitudes in the heavy-quark limit [1.2]. The underlying idea is to exploit the presence of a large scale, i.e., the fact that $m_{b} \gg \Lambda_{\mathrm{QCD}}$. In order to disentangle the physics associated with these two scales, we factorize and compute hard contributions to the decay amplitudes arising from gluons with virtuality of order $m_{b}$, and parameterize soft and collinear contributions. Considering the cartoon in Fig. 1, we denote by $M_{1}$ the meson that absorbs the spectator quark of the $B$ meson, and by $M_{2}$ the meson at the upper vertex, to which we refer as the "emission particle". We find that at leading power in $\Lambda_{\mathrm{QCD}} / m_{b}$ all long-distance contributions to the decay amplitudes can be factorized into semileptonic form factors and meson light-cone distribution amplitudes, which are much simpler quantities than the nonleptonic amplitudes themselves. A graphical representation of the resulting "factorization formula" is shown in Fig. 2. The physical picture underlying factorization is color transparency [3,4]. If the emission particle is a light meson, its constituents carry large energy of order $m_{b}$ and are nearly collinear. Soft gluons coupling to this system see only its net zero color charge and hence decouple. Interactions with the color dipole of the small $q \bar{q}$-pair are power suppressed in the heavyquark limit.

For $B$ decays into final states containing a heavy charm meson and a light meson, the factorization formula takes the form

$$
\begin{aligned}
\left\langle D^{(*)+}\right. & \left.L^{-}\left|O_{i}\right| \bar{B}_{d}\right\rangle=\sum_{j} F_{j}^{B \rightarrow D^{(*)}} f_{L} \\
& \times \int_{0}^{1} \mathrm{~d} u T_{i j}^{I}(u) \Phi_{L}(u)+\mathcal{O}\left(\frac{\Lambda_{\mathrm{QCD}}}{m_{b}}\right),
\end{aligned}
$$

where $O_{i}$ is an operator in the effective weak Hamiltonian (11), $F_{j}^{B \rightarrow D^{(*)}}$ are transition form factors (evaluated at $\left.q^{2}=m_{L}^{2} \approx 0\right), f_{L}$ and $\Phi_{L}$ are the decay constant and leading-twist lightcone distribution amplitude of the light meson, and $T_{i j}^{I}$ are process-dependent hard-scattering kernels. For decays into final states containing

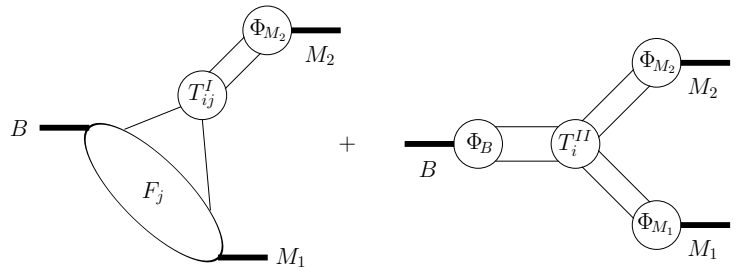

Figure 2. QCD factorization in the heavy-quark limit. The second term is power suppressed for $B \rightarrow D \pi$, but must be kept for decays with two light mesons in the final state, such as $B \rightarrow \pi K$. Contributions not shown (such as weak annihilation graphs) are power suppressed.

two light mesons there is a second type of contribution to the factorization formula, which involves a hard interaction with the spectator quark in the $B$ meson. This is shown by the second graph in Fig. 2. Below we focus first on $\bar{B} \rightarrow D^{(*)} L$ decays (with $L$ a light meson), where this second term is power suppressed and can be neglected. Decays into two light final-state mesons are more complicated [1.5] and will be discussed briefly in Sect. 4 .

The factorization formula for nonleptonic decays provides a model-independent basis for the analysis of these processes in an expansion in powers and logarithms of $\Lambda_{\mathrm{QCD}} / m_{b}$. At leading power, but to all orders in $\alpha_{s}$, the decay amplitudes assume the factorized form shown in (2). Having such a formalism based on power counting in $\Lambda_{\mathrm{QCD}} / m_{b}$ is of great importance to the theoretical description of hadronic weak decays, since it provides a well-defined limit of QCD in which these processes admit a rigorous, theoretical description. (For instance, the possibility to compute systematically $\mathcal{O}\left(\alpha_{s}\right)$ corrections to "naive factorization", which emerges as the leading term in the heavy-quark limit, solves the old problem of renormalization-scale and scheme dependences of nonleptonic amplitudes.) The usefulness of this new scheme may be compared with the usefulness of the heavy-quark effective theory for the analysis of exclusive semileptonic decays of heavy mesons, or of the heavy-quark expansion for the analysis of inclusive decay rates. In all three cases, it is the fact that hadronic uncer- 
tainties can be eliminated up to power corrections in $\Lambda_{\mathrm{QCD}} / m_{b}$ that has advanced our ability to control theoretical errors.

It must be stressed, however, that we are just beginning to explore the theory of nonleptonic $B$ decays. Some important conceptual problems remain to be better understood. In the next few years it will be important to further develop the approach. This should include an all-orders proof of factorization at leading power, the development of a formalism for dealing with power corrections to factorization, understanding the lightcone structure of heavy mesons, and understanding the relevance (or irrelevance) of Sudakov form factors. Also, we must gauge the accuracy of the approach by learning about the magnitude of corrections to the heavy-quark limit from extensive comparisons of theoretical predictions with data.

As experience with previous heavy-quark expansions has shown, this is going to be a long route. Yet, already we have obtained important insights. Before turning to specific applications, let me mention three points here:

1. Corrections to "naive factorization" (usually called "nonfactorizable effects") are process dependent, in contrast with a basic assumption underlying models of "generalized factorization".

2. The physics of nonleptonic decays is both rich and complicated. There may, in general, be an interplay of several small parameters (Wilson coefficients, CKM factors, $1 / N_{c}$, etc.) in addition to the small parameter $\Lambda_{\mathrm{QCD}} / m_{b}$ relevant to QCD factorization. Also, several notso-well-known input parameters (e.g., heavy-tolight form factors and light-cone distribution amplitudes) introduce numerical uncertainties.

3 . Strong rescattering phases arising from finalstate interactions are suppressed in the heavyquark limit. More precisely, the imaginary parts of nonleptonic decay amplitudes are suppressed by at least one power of $\alpha_{s}\left(m_{b}\right)$ or $\Lambda_{\mathrm{QCD}} / m_{b}$. At leading power, the phases are calculable from the imaginary parts of the hard-scattering kernels in the factorization formula. A generic consequence is that direct $\mathrm{CP}$ asymmetries in hadronic $B$ decays are suppressed in the heavy-quark limit, except for cases where for some reason the real part of the decay amplitude is also suppressed.

\section{APPLICATIONS TO $\bar{B}_{d} \rightarrow D^{(*)+} L^{-}$ DECAYS}

Our result for the nonleptonic $\bar{B}_{d} \rightarrow D^{(*)+} L^{-}$ decay amplitudes (with $L$ a light meson) can be compactly expressed in terms of the matrix elements of a "transition operator"

$\mathcal{T}=\frac{G_{F}}{\sqrt{2}} V_{u d}^{*} V_{c b}\left[a_{1}(D L) Q_{V}-a_{1}\left(D^{*} L\right) Q_{A}\right]$,

where the hadronic matrix elements of the operators $Q_{V}=\bar{c} \gamma^{\mu} b \otimes \bar{d} \gamma_{\mu}\left(1-\gamma_{5}\right) u$ and $Q_{A}=$ $\bar{c} \gamma^{\mu} \gamma_{5} b \otimes \bar{d} \gamma_{\mu}\left(1-\gamma_{5}\right) u$ are understood to be evaluated in factorized form. Eq. (3) defines the quantities $a_{1}\left(D^{(*)} L\right)$, which include the leading "nonfactorizable" corrections, in a renormalizationgroup invariant way. To leading power in $\Lambda_{\mathrm{QCD}} / m_{b}$ these quantities should not be interpreted as phenomenological parameters (as is usually done), because they are dominated by hard gluon exchange and thus calculable in QCD. At next-to-leading order in $\alpha_{s}$ we obtain [2]

$$
\begin{aligned}
& a_{1}\left(D^{(*)} L\right)=\bar{C}_{1}\left(m_{b}\right)+\frac{\bar{C}_{2}\left(m_{b}\right)}{N_{c}}[1+ \\
& \left.+\frac{C_{F} \alpha_{s}\left(m_{b}\right)}{4 \pi} \int_{0}^{1} \mathrm{~d} u F(u, \pm z) \Phi_{L}(u)\right],
\end{aligned}
$$

where $z=m_{c} / m_{b}, \bar{C}_{i}\left(m_{b}\right)$ are the so-called "renormalization-scheme independent" Wilson coefficients, and the upper (lower) sign in the second argument of the function $F(u, \pm z)$ refers to a $D\left(D^{*}\right)$ meson in the final state. The exact expression for this function is known but not relevant here. Note that the coefficients $a_{1}(D L)$ and $a_{1}\left(D^{*} L\right)$ are nonuniversal, i.e., they are explicitly dependent on the nature of the final-state mesons. Politzer and Wise have computed the "nonfactorizable" vertex corrections to the decay rate ratio of $\bar{B}_{d} \rightarrow D^{+} \pi^{-}$and $\bar{B}_{d} \rightarrow D^{*+} \pi^{-}[\bar{b}$. This requires the symmetric part (with respect to $u \leftrightarrow 1-u)$ of the difference $F(u, z)-F(u,-z)$. We agree with their result.

The expressions for the decay amplitudes obtained by evaluating the hadronic matrix elements of the transition operator $\mathcal{T}$ involve products of CKM matrix elements, light-meson decay constants, $\bar{B} \rightarrow D^{(*)}$ transition form factors, 
and the QCD parameters $a_{1}\left(D^{(*)} L\right)$. A numerical analysis shows that $\left|a_{1}\right|=1.055 \pm 0.025$ for the decays considered below [2]. Below we will use this as our central value.

\subsection{Tests of factorization}

A particularly clean test of our predictions is obtained by relating the $\bar{B}_{d} \rightarrow D^{*+} L^{-}$decay rates to the differential semileptonic $\bar{B}_{d} \rightarrow$ $D^{*+} l^{-} \nu$ decay rate evaluated at $q^{2}=m_{L}^{2}$. In this way the parameters $\left|a_{1}\right|$ can be measured directly, since [3]

$$
\begin{aligned}
& \frac{\Gamma\left(\bar{B}_{d} \rightarrow D^{*+} L^{-}\right)}{d \Gamma\left(\bar{B}_{d} \rightarrow D^{*+} l^{-} \bar{\nu}\right) /\left.d q^{2}\right|_{q^{2}=m_{L}^{2}}} \\
& \quad=6 \pi^{2}\left|V_{u d}\right|^{2} f_{L}^{2}\left|a_{1}\left(D^{*} L\right)\right|^{2} .
\end{aligned}
$$

With our result for $a_{1}$ this relation becomes a prediction based on first principles of QCD. This is to be contrasted with the usual interpretation of this formula, where $a_{1}$ plays the role of a phenomenological parameter that is fitted from data.

Using results reported by the CLEO Collaboration [7], we find

$$
\begin{aligned}
\left|a_{1}\left(D^{*} \pi\right)\right| & =1.08 \pm 0.07, \\
\left|a_{1}\left(D^{*} \rho\right)\right| & =1.09 \pm 0.10, \\
\left|a_{1}\left(D^{*} a_{1}\right)\right| & =1.08 \pm 0.11,
\end{aligned}
$$

in good agreement with our prediction. It is reassuring that the data show no evidence for large power corrections to our results. However, a further improvement in the experimental accuracy would be desirable in order to become sensitive to process-dependent, nonfactorizable effects.

\subsection{Predictions for class-I amplitudes}

We now consider a larger set of so-called class-I decays of the form $\bar{B}_{d} \rightarrow D^{(*)+} L^{-}$, all of which are governed by the transition operator (3). In Tab. 1 we compare the QCD factorization predictions with experimental data. As previously we work in the heavy-quark limit, i.e., our predictions are model independent up to corrections suppressed by at least one power of $\Lambda_{\mathrm{QCD}} / m_{b}$. There is good agreement between our predictions and the data within experimental errors, which however are still large. It would be desirable to reduce these errors to the percent level. (Note that
Table 1

Model-independent predictions for the branching ratios (in units of $\left.10^{-3} \times\left(\left|a_{1}\right| / 1.05\right)^{2}\right)$ of $\bar{B}_{d} \rightarrow$ $D^{(*)+} L^{-}$decays in the heavy-quark limit.

\begin{tabular}{|l|c|c|}
\hline Decay mode & Theory (HQL) & PDG [8] \\
\hline $\bar{B}_{d} \rightarrow D^{+} \pi^{-}$ & $3.27 \times\left[F_{+} / 0.6\right]^{2}$ & $3.0 \pm 0.4$ \\
$\bar{B}_{d} \rightarrow D^{+} K^{-}$ & $0.25 \times\left[F_{+} / 0.6\right]^{2}$ & - \\
$\bar{B}_{d} \rightarrow D^{+} \rho^{-}$ & $7.64 \times\left[F_{+} / 0.6\right]^{2}$ & $7.9 \pm 1.4$ \\
$\bar{B}_{d} \rightarrow D^{+} K^{*-}$ & $0.39 \times\left[F_{+} / 0.6\right]^{2}$ & - \\
$\bar{B}_{d} \rightarrow D^{+} a_{1}^{-}$ & $7.76 \times\left[F_{+} / 0.6\right]^{2}$ & $6.0 \pm 3.3$ \\
\hline $\bar{B}_{d} \rightarrow D^{*+} \pi^{-}$ & $3.05 \times\left[A_{0} / 0.6\right]^{2}$ & $2.8 \pm 0.2$ \\
$\bar{B}_{d} \rightarrow D^{*+} K^{-}$ & $0.22 \times\left[A_{0} / 0.6\right]^{2}$ & - \\
$\bar{B}_{d} \rightarrow D^{*+} \rho^{-}$ & $7.59 \times\left[A_{0} / 0.6\right]^{2}$ & $6.7 \pm 3.3$ \\
$\bar{B}_{d} \rightarrow D^{*+} K^{*-}$ & $0.40 \times\left[A_{0} / 0.6\right]^{2}$ & - \\
$\bar{B}_{d} \rightarrow D^{*+} a_{1}^{-}$ & $8.53 \times\left[A_{0} / 0.6\right]^{2}$ & $13.0 \pm 2.7$ \\
\hline
\end{tabular}

we have not attempted to adjust the semileptonic form factors $F_{+}^{\bar{B} \rightarrow D}$ and $A_{0}^{\bar{B} \rightarrow D^{*}}$ entering our results so as to obtain a best fit to the data.)

The observation that the experimental data on class-I decays into heavy-light final states show good agreement with our predictions may be taken as (circumstantial) evidence that in these decays there are no unexpectedly large power corrections. In our recent work [2] we have addressed the important question of power corrections theoretically by providing estimates for two sources of power-suppressed effects: weak annihilation and spectator interactions. A complete account of power corrections to the heavy-quark limit can at present not be performed in a systematic way, since these effects are no longer dominated by hard gluon exchange. However, we believe that our estimates are nevertheless instructive.

We parameterize the annihilation contribution to the $\bar{B}_{d} \rightarrow D^{+} \pi^{-}$decay amplitude in terms of an amplitude $A$ such that $\mathcal{A}\left(\bar{B}_{d} \rightarrow D^{+} \pi^{-}\right)=$ $T+A$, where $T$ is the "tree topology", which contains the dominant factorizable contribution. We find that $A / T \sim 0.04$. We have also obtained an estimate of nonfactorizable spectator interactions, which are part of $T$, finding that $T_{\text {spec }} / T_{\text {lead }} \sim-0.03$. In both cases, the results exhibit the expected linear power suppression in 
the heavy-quark limit. We conclude that the typical size of power corrections in class-I decays into heavy-light final states is at the level of $10 \%$ or less, and thus our predictions for the values and the near universality of the parameters $a_{1}$ governing these decay modes appear robust.

\section{QCD FACTORIZATION IN CHARM- LESS HADRONIC B DECAYS}

The observation of rare charmless $B$ decays into $\pi K$ and $\pi \pi$ final states has resulted in a large amount of theoretical and phenomenological work that attempts to interpret these observations in terms of the factorization approximation, or in terms of general parameterizations of the decay amplitudes. A detailed understanding of these amplitudes would help us to pin down the value of the CKM angle $\gamma=\arg \left(V_{u b}^{*}\right)$ using only data on $\mathrm{CP}$-averaged branching fractions. Here we briefly summarize the most important consequences of the QCD factorization approach for the $\pi K$ and $\pi \pi$ final states $[5$.

To leading order in an expansion in powers of $\Lambda_{\mathrm{QCD}} / m_{b}$, the $B \rightarrow \pi K$ matrix elements obey the factorization formula shown in Fig. 2 :

$$
\begin{aligned}
\left\langle\pi K\left|O_{i}\right| B\right\rangle & =F_{+}^{B \rightarrow \pi} f_{K} T_{K, i}^{I} * \Phi_{K} \\
& +F_{+}^{B \rightarrow K} f_{\pi} T_{\pi, i}^{I} * \Phi_{\pi} \\
& +f_{B} f_{K} f_{\pi} T_{i}^{I I} * \Phi_{B} * \Phi_{K} * \Phi_{\pi},
\end{aligned}
$$

where the $*$-products imply an integration over the light-cone momentum fractions of the constituent quarks inside the mesons. Our results are based on hard-scattering kernels including all corrections of order $\alpha_{s}$. Compared to our previous discussion of $B \rightarrow \pi \pi$ decays [1], the present analysis incorporates three new ingredients: the matrix elements of electroweak penguin operators (for $\pi K$ modes), hard-scattering kernels for asymmetric light-cone distributions, and the complete set of "chirally enhanced" $1 / m_{b}$ corrections. The second and third items have not been considered in other generalizations of Ref. [1] to the $\pi K$ final states 9.10$]$. The third one, in particular, is essential for estimating some of the theoretical uncertainties of the approach. For completeness, we note that the predictions from QCD factorization differ in essential aspects from those obtained in the conventional hard-scattering approach [11].

Following Ref. [1], we have obtained the coefficients $a_{i}(\pi K)$ (with $i=1, \ldots, 10$ ) of the effective, factorized "transition operator" defined analogously to the case of $B \rightarrow \pi \pi$ decays, but augmented by coefficients related to electroweak penguin contributions. Chirally enhanced corrections arise from twist-3 two-particle light-cone distribution amplitudes, whose normalization involves the quark condensate. The relevant parameter, $2 \mu_{\pi} / m_{b}=-4\langle\bar{q} q\rangle /\left(f_{\pi}^{2} m_{b}\right)$, is formally of order $\Lambda_{\mathrm{QCD}} / m_{b}$, but large numerically. The coefficients $a_{6}$ and $a_{8}$ are multiplied by this parameter. There are also additional chirally enhanced corrections to the spectator-interaction term in (7), which turn out to be the more important effect. These latter corrections involve a logarithmically divergent integral, which violates factorization. For instance, for matrix elements of $V-A$ operators the hard spectator interaction is proportional to $(\bar{u} \equiv 1-u, \bar{v} \equiv 1-v)$

$\int_{0}^{1} \frac{d u}{\bar{u}} \frac{d v}{\bar{v}} \Phi_{K}(u)\left(\Phi_{\pi}(v)+\frac{2 \mu_{\pi}}{m_{b}} \frac{\bar{u}}{u}\right)$

when the spectator quark goes to the pion. (Here we used that the twist-3 distribution amplitudes can be taken to be the asymptotic ones when one neglects twist-3 corrections without the chiral enhancement.) The divergence of the $v$-integral in the second term as $\bar{v} \rightarrow 0$ implies that it is dominated by soft gluon exchange between the spectator quark and the quarks that form the kaon. We therefore treat the divergent integral $X=\int_{0}^{1}(d v / \bar{v})$ as an unknown parameter, which may in principle be complex owing to soft rescattering in higher orders. In our numerical analysis we set $X=\ln \left(m_{B} / 0.35 \mathrm{GeV}\right)+r$, where $r$ is chosen randomly inside a circle in the complex plane of radius 3 ("realistic") or 6 ("conservative"). Our results also depend on the $B$-meson parameter [1] $\lambda_{B}$, which we vary between 0.2 and $0.5 \mathrm{GeV}$. Finally, there is in some cases a nonnegligible dependence of the coefficients $a_{i}(\pi K)$ on the renormalization scale, which we vary between $m_{b} / 2$ and $2 m_{b}$.

We take $\left|V_{u b} / V_{c b}\right|=0.085$ and $m_{s}(2 \mathrm{GeV})=$ 

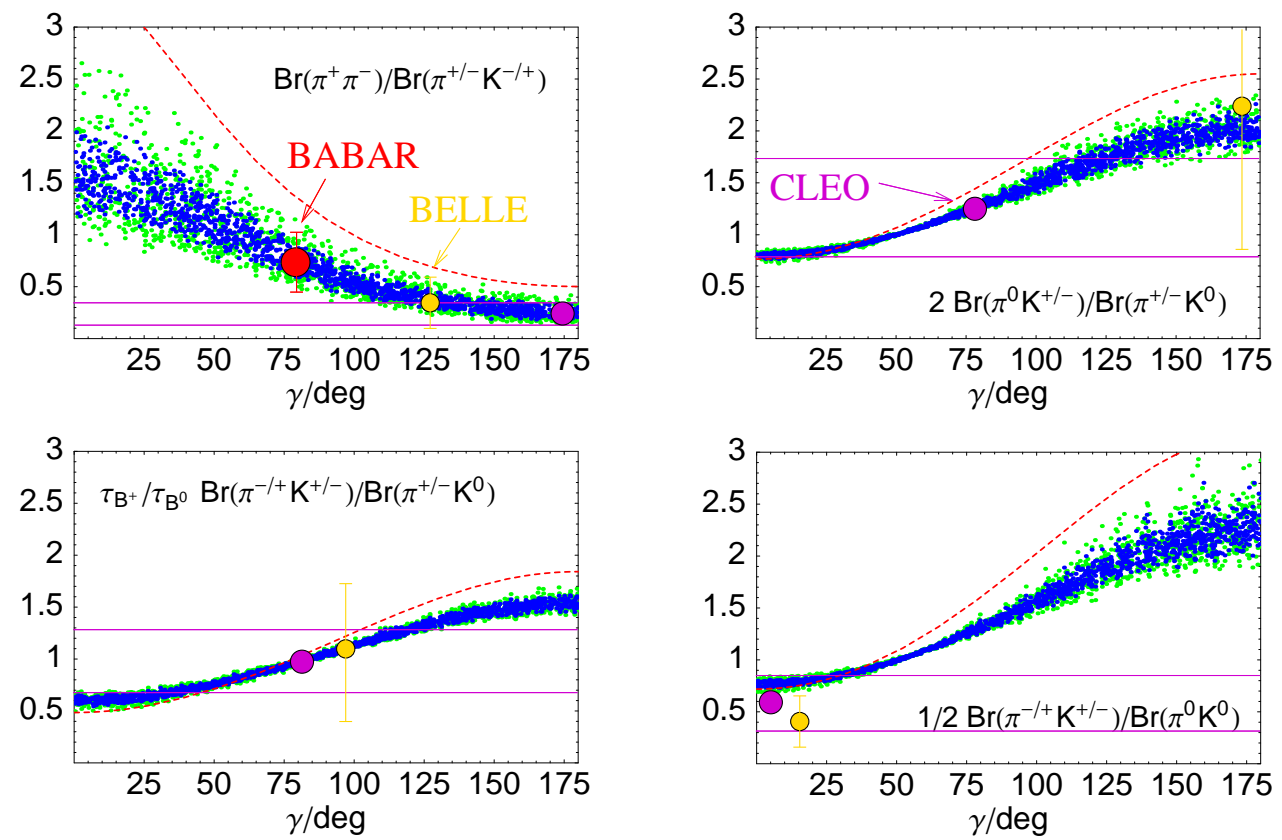

Figure 3. Ratios of CP-averaged $B \rightarrow \pi K$ and $\pi \pi$ decay rates. The scattered points cover a realistic (dark) and conservative (light) variation of input parameters. The dashed curve is the result obtained using "naive factorization".

$110 \mathrm{MeV}$ as fixed input to our analysis, noting that ultimately these Standard Model parameters, along with the CP-violating phase $\gamma$, might be extracted from a simultaneous fit to the $B \rightarrow$ $\pi K$ and $B \rightarrow \pi \pi$ decay rates. We now summarize our main results.

\subsection{Results on SU(3) breaking}

Bounds on $\gamma$ derived from ratios of $\mathrm{CP}$ averaged $B \rightarrow \pi K$ decay rates 12, 13], as well as the determination of $\gamma$ using the method of Ref. [14], rely on an estimate of $\mathrm{SU}(3)$ flavorsymmetry violations. We find that "nonfactorizable" SU(3)-breaking effects (i.e., effects not accounted for by the different decay constants and form factors of pions and kaons in the conventional factorization approximation) do not exceed the level of a few percent.

\subsection{Ratios of CP-averaged rates}

The approach discussed here allows us to calculate the amplitudes for $B \rightarrow \pi K$ and $B \rightarrow \pi \pi$ decays in terms of form factors and light-cone distribution amplitudes. We focus on decays whose branching ratios have already been measured. Since the relevant form factor $F_{+}^{B \rightarrow \pi}(0)$ is not well known, we consider only ratios of $\mathrm{CP}$ averaged branching ratios. We display these as functions of the weak phase $\gamma$ in Fig. 3. For comparison, we show the data on the various ratios obtained using results reported by the CLEO Collaboration $[15]$. We also indicate the very recent results reported by the BaBar and Belle Collaborations at the ICHEP2000 Conference [16]. From the plots it is apparent that the corrections with respect to the conventional factorization approximation are significant (and important to reduce the renormalization-scale dependence). Despite this fact, we find that the qualitative pattern emerging for the set of $B \rightarrow \pi K$ and $\pi \pi$ decay modes is similar to that in conventional factorization. In particular, the penguin-tree interference is constructive (destructive) in $B \rightarrow \pi^{+} \pi^{-}$ $\left(B \rightarrow \pi^{-} K^{+}\right)$decays if $\gamma<90^{\circ}$. Taking the currently favored range $\gamma=(60 \pm 20)^{\circ}$, we find the 
following robust predictions:

$$
\begin{array}{rr}
\frac{\operatorname{Br}\left(\pi^{+} \pi^{-}\right)}{\operatorname{Br}\left(\pi^{\mp} K^{ \pm}\right)}=0.5-1.9 \quad[0.25 \pm 0.10], \\
\frac{2 \operatorname{Br}\left(\pi^{0} K^{ \pm}\right)}{\operatorname{Br}\left(\pi^{ \pm} K^{0}\right)}=0.9-1.3 \quad[1.27 \pm 0.47], \\
\frac{\tau_{B^{+}}}{\tau_{B^{0}}} \frac{\operatorname{Br}\left(\pi^{\mp} K^{ \pm}\right)}{\operatorname{Br}\left(\pi^{ \pm} K^{0}\right)}=0.6-1.0 \quad[1.00 \pm 0.30], \\
\frac{\operatorname{Br}\left(\pi^{\mp} K^{ \pm}\right)}{2 \operatorname{Br}\left(\pi^{0} K^{0}\right)}=0.9-1.4 \quad[0.59 \pm 0.27] .
\end{array}
$$

The first ratio is clearly in disagreement with current CLEO data [15] shown in square brackets. However, there is good agreement with the recent results reported by BaBar $(0.74 \pm 0.29)$ and Belle $(0.36 \pm 0.26)$ 16.

The near equality of the second and fourth ratios in (9) is a consequence of isospin symmetry 13. We find $\operatorname{Br}\left(B \rightarrow \pi^{0} K^{0}\right)=(4.5 \pm 2.5) \times$ $10^{-6} \times\left[F_{+}^{B \rightarrow \pi}(0) / 0.3\right]^{2}$ almost independently of $\gamma$. This is three time smaller than the central value reported by CLEO, $\left(14.6_{-5.1-3.3}^{+5.9+2.4}\right) \times 10^{-6}$, and four times smaller than the central value reported by BELLE, $\left(21.0_{-7.8-2.3}^{+9.3+2.5}\right) \times 10^{-6}$. It will be interesting to follow how the comparison between data and theory develops as the data become more precise.

\subsection{CP asymmetry in $B \rightarrow \pi^{+} \pi^{-}$decay}

The stability of the prediction for the $B \rightarrow$ $\pi^{+} \pi^{-}$amplitude suggests that the CKM angle $\alpha$ can be extracted from the time-dependent mixing-induced $\mathrm{CP}$ asymmetry in this decay mode, without using an isospin analysis. Figure 1 displays the coefficient $S$ of $-\sin \left(\Delta m_{B_{d}} t\right)$ in the time-dependent asymmetry as a function of $\sin (2 \alpha)$ for $\sin (2 \beta)=0.75$. For some values of $S$ there is a two-fold ambiguity (assuming all angles are between $0^{\circ}$ and $180^{\circ}$ ). A consistency check of the approach could be obtained, in principle, from the coefficient of the $\cos \left(\Delta m_{B_{d}} t\right)$ term, which is given by the direct $\mathrm{CP}$ asymmetry in this decay.

\section{SUMMARY AND OUTLOOK}

With the recent commissioning of the $B$ factories and the planned emphasis on heavy-flavor physics in future collider experiments, the role of



Figure 4. Mixing-induced CP asymmetry in $B \rightarrow$ $\pi^{+} \pi^{-}$decays. The lower band refers to values $45^{\circ}<\alpha<135^{\circ}$, the upper one to $\alpha<45^{\circ}$ (right) or $\alpha>135^{\circ}$ (left). We assume $\alpha, \beta, \gamma \in\left[0,180^{\circ}\right]$.

$B$ decays in providing fundamental tests of the Standard Model and potential signatures of New Physics will continue to grow. In many cases the principal source of systematic uncertainty is a theoretical one, namely our inability to quantify the nonperturbative QCD effects present in these decays. This is true, in particular, for almost all measurements of direct $\mathrm{CP}$ violation. Our work provides a rigorous framework for the evaluation of strong-interaction effects for a large class of exclusive, two-body nonleptonic decays of $B$ mesons. It gives a well-founded field-theoretic basis for phenomenological studies of exclusive hadronic $B$ decays and a formal justification for the ideas of factorization.

We believe that the factorization formula (2) and its generalization to decays into two light mesons will form a useful basis for future phenomenological studies of nonleptonic $B$ decays. We stress, however, that a considerable amount of conceptual work remains to be completed. Theoretical investigations along the lines discussed here should be pursued with vigor. We are confident that, ultimately, this research will result in a theory of nonleptonic $B$ decays.

\section{Acknowledgments}

It is a pleasure to thank the organizers of the Ferrara Conference for a splendid meeting and generous support. I am grateful to Martin Beneke, Gerhard Buchalla and Chris Sachra- 
jda for an ongoing collaboration on the subject of this talk. This work was supported in part by the National Science Foundation.

\section{REFERENCES}

1. M. Beneke, G. Buchalla, M. Neubert and C.T. Sachrajda, Phys. Rev. Lett. 83, 1914 (1999).

2. M. Beneke, G. Buchalla, M. Neubert and C.T. Sachrajda, Preprint hep-ph/0006124, to appear in Nucl. Phys. B.

3. J.D. Bjorken, Nucl. Phys. B (Proc. Suppl.) 11, 325 (1989).

4. M.J. Dugan and B. Grinstein, Phys. Lett. B 255, 583 (1991).

5. M. Beneke, G. Buchalla, M. Neubert and C.T. Sachrajda, Preprint hep-ph/0007256.

6. H.D. Politzer and M.B. Wise, Phys. Lett. B 257, 399 (1991).

7. J.L. Rodriguez (CLEO Collaboration), Proceedings of the 2nd International Conference on $B$ Physics and $C P$ Violation, edited by T.E. Browder et al. (World Scientific, Singapore, 1998), pp. 124 (hep-ex/9801028).

8. C. Caso et al. (Particle Data Group), Eur. Phys. J. C 3, 1 (1998).

9. D. Du, D. Yang and G. Zhu, Phys. Lett. B 488, 46 (2000).

10. T. Muta, A. Sugamoto, M. Yang and Y. Yang, Phys. Rev. D 62, 094020 (2000).

11. Y.Y. Keum, H.-n. Li and A.I. Sanda, Preprints hep-ph/0004004 and 0004173.

12. R. Fleischer and T. Mannel, Phys. Rev. D 57, 2752 (1998).

13. M. Neubert and J.L. Rosner, Phys. Lett. B 441, 403 (1998);

M. Neubert, JHEP 02, 014 (1999).

14. M. Neubert and J.L. Rosner, Phys. Rev. Lett. 81, 5076 (1998);

M. Neubert, Nucl. Phys. B (Proc. Suppl.) 86, 477 (2000).

15. D. Cronin-Hennessy et al. (CLEO Collaboration), Preprint hep-ex/0001010.

16. Talks by presented by T.J. Champion (BaBar Collaboration) and P. Chang (Belle Collaboration) at the XXXth International Conference on High-Energy Physics, Osaka, Japan, 27 July - 2 August, 2000. 